\title{
Síndrome del hombre maltratado y la violencia intrafamiliar.
}

\author{
Syndrome of the battered man and with domestic violence
}

Viviana Barros Carvajal ${ }^{1}$, Edmundo Chóez Chiliquinga. ${ }^{2}$, \& Fernanda Zambrano Pérez ${ }^{3}$

Recibido: 10-02-2019 / Revisado: 15-02-209 /Aceptado: 04-03-2019/ Publicado: 14-06-2019

\begin{abstract}
.
DOI: https://doi.org/10.33262/cienciadigital.v3i1.1.365

This document analyzes the characteristics of the man assaulted, the type of violence of which they are victims, the characteristics of the aggressor and the injuries they suffer, to give a complete perspective of domestic violence in Ecuador. Objectives. Identify the presence of the Syndrome of the mistreated man, the characteristics of his aggressors and his relation with the domestic violence. Methods. - Observational, descriptive, cross-sectional investigation. Results.-There 442 cases were married men, with an average of 30 to 34 years of age. The main aggressor was his wife. The $76.8 \%$ of the victims suffered domestic violence before, most of them had 3 days of legal medical incapacity. The $72.9 \%$ are head and face injuries, emitted by blunt objects in a $76.5 \%$. Conclusions.-The $77 \%$ of men have suffered violence before, only a small percentage have reported it. There is a relationship between the syndrome of battered man and domestic violence.
\end{abstract}

Keywords: domestic, violence, battered, men

\section{Resumen.}

En este documento se analiza las características del hombre agredido, el tipo de violencia del que son víctimas, las características de la agresora y las lesiones que sufren, para dar una perspectiva completa de la violencia intrafamiliar en el Ecuador.

\footnotetext{
${ }^{1}$ Universidad Central del Ecuador. Correo-e: mvbcmedi7@ hotmail.com

2 Universidad Central del Ecuador. Correo-e: edmundo choez@hotmail.com

${ }^{3}$ Universidad Central del Ecuador. Correo-e: a15_fernanda@hotmail.com
} 
Objetivos.-Identificar la presencia del Síndrome del hombre maltratado, las características de sus agresoras y su relación con la violencia intrafamiliar. Métodos.Observacional, descriptiva de corte transversal. Resultados.-Los 442 casos fueron hombres casados, con un promedio de 30 a 34 años. La principal agresora fue su esposa. El 76,8\% de las víctimas sufrió violencia intrafamiliar anteriormente que presentaron 3 días de incapacidad médico legal, el 72,9\% son lesiones a nivel de cabeza y cara, emitidas por objetos contundentes en un 76,5\%. Conclusiones.-El 77\% de hombres ha sufrido violencia anteriormente, solo un pequeño porcentaje lo ha denunciado, por lo tanto, existe relación entre el Síndrome del hombre maltratado y la violencia intrafamiliar.

Palabras claves: Violencia, intrafamiliar, hombre, maltrato

\section{Introducción.}

El tipo de violencia más representativo a nivel nacional es la violencia intrafamiliar, sin embargo, durante años se ha considerado como generador de violencia al hombre, dejando de lado que tanto hombres como mujeres pueden ser víctimas de malos tratos, por parte de sus cónyuges, o parejas sentimentales; la literatura revisada para el planteamiento del presente estudio trata en su mayoría sobre la violencia hacia la mujer., teniendo muy poca escasos documentos acerca de la violencia hacia el hombre. El maltrato de las parejas hacia el hombre muchas veces se ha conjugado bajo amenazas de abandono y separándolo de su familia como son sus hijos y sus bienes materiales, muchas de ellas no se adjudican un compromiso de culpabilidad y atribuyen su falta a su pareja, el hombre en este sentido toma la culpa como suya y siente que es justo el maltrato que está recibiendo. Muchos de ellos no se separan de sus mujeres por sus hijos, por el temor de perderlos y ante todo que su madre los coloque en su contra y termine la buena relación que existe entre ellos. La violencia hacia el hombre es oculta y subregistrada en nuestro país por lo que se realiza este estudio para el conocimiento de la población ecuatoriana.

La violencia intrafamiliar, por definición, son todas las situaciones o formas de abuso de poder o maltrato (físico o psicológico) de un miembro de la familia sobre otro que se desarrollan en el contexto de las relaciones familiares y que ocasionan diversos niveles de daño a las víctimas de esos abusos, por ende es una situación "multicausal" donde factores sociales, individuales, comunitarios conjuntamente con edad, sexo, nivel económico, situación laboral, nivel de educación, uso de drogas entre otras sustancias, presenciar o sufrir maltrato físico en la niñez y todas estas causas pueden incurrir en violencia, no siempre determinan la misma, sin embargo, cada uno de estos factores puede predeterminar un comportamiento violento o el llegar a ser objeto de violencia. Según la Organización Mundial de la Salud la violencia en la familia es la agresión física, psicológica o sexual cometida por el esposo/a o conviviente, abuelos, padres, hijos, hermanos, parientes civiles u otros 
familiares. También comprende a los tutores o encargados de la custodia. Afecta a todas las familias sin distinción de raza, edad, educación o condiciones socioeconómicas.

El síndrome del hombre maltratado es un concepto creado por Suzanne K. Steinmetz el mismo que se refiere a una condición física y psicológica que presentan las víctimas de violencia y abusos (usualmente de manera persistente) proveniente de sus parejas. Los varones que se encuentran ante esta situación reaccionan de dos formas, la primera, optar por el silencio o la segunda, expresarlo de manera pública, los que salen a la luz desean superar su ocultamiento. Los hombres víctimas de la violencia por parte de la pareja, pueden experimentar lesiones graves como extremidades fracturadas, heridas por armas blancas, mordeduras, excoriaciones, laceraciones, agresiones verbales, intimidación psicológica y emocional. En consecuencia, las víctimas masculinas pueden rechazar el examen físico realizado por médicos legistas, el apoyo de profesionales de la salud y en general de los servicios de salud; como resultado, muchos hombres temen buscar ayuda legal, por el hecho de que los consideren los agresores cuando ellos son los agredidos, ante todo, cuando es en defensa propia.

\section{Metodologia.}

El estudio configura un diseño de carácter: observacional por cuanto la información se obtuvo de los expedientes de la Fiscalía de Pichincha; descriptivo, transversal, la información obtenida se realiza en un determinado período de tiempo. Este diseño permite afirmar la presencia de Síndrome de hombre maltratado, registrados en los expedientes de la Unidad de Atención de Peritaje Integral de Quitumbe y en la Unidad de Delitos Flagrantes del departamento de Medicina Legal de la ciudad de Quito, en el período de enero 2016 hasta diciembre del 2017, de estos documentos se extrajeron las características del agredido, y del agresor, así como las lesiones presentes, mecanismo del trauma, objetos que usó la pareja agresora, sin olvidar el tiempo de incapacidad médico legal.

Se definió una población de 442 casos de hombres agredidos en el período comprendido entre enero del 2016 a diciembre del 2017; se decidió realizar el estudio a toda la población, observando los criterios de inclusión y exclusión.

Entre los criterios de inclusión tenemos: Hombres agredidos con informe pericial examinados por un médico forense en el departamento de Medicina Legal de la Unidades de Atención de Peritaje Integral de Quitumbe y Unidad de Flagrancia de la ciudad de Quito durante el año 2016 y el año 2017; reportes de hombres mayores de 18 años en adelante.

Los criterios de exclusión son: Hombres agredidos que no cuenten con informe pericial en el departamento de Medicina Legal de la Unidades de Atención de Peritaje Integral de Quitumbe y Unidad de Flagrancia de la ciudad de Quito durante el año 2016 - 2017; hombres agredidos por otro personal del núcleo familiar. 


\section{Resultados.}

El estudio analizó los 442 expedientes (formulario de la Fiscalía General del Estado) de Medicina Legal desde enero de 2016 a diciembre de 2017.

\section{- Características de los hombres agredidos (maltratados).}

\section{Grupos de edad de hombres maltratados. Fiscalía de Pichincha, 2016-2017}

Se elaboraron grupos de edad; de las 442 víctimas registradas, el promedio fue de 35,5 años, con una desviación estándar de +/- 10,9 años con límites de edad entre 18 y 75; en relación a la clasificación por grupos de edad el $25,6 \%$ corresponde al grupo de 30 a 34 años, mientras que el 18,4\% constituyen las víctimas de 25 a 29 años, el 18,1\% corresponde al grupo de 45 a 59 años y el 16,1\% corresponde al grupo etario de 35 a 39 años, el resto de grupos etarios representan menos del $10 \%$ cada uno.

Estado civil de las víctimas. Fiscalía de Pichincha, 2016-2017.

Con respecto al estado civil de las víctimas, el $40 \%$ fueron casados, $30 \%$ de los casos unión libre, el $22 \%$ fueron solteros y el $8 \%$ divorciado o separado.

\section{Nivel educativo de las víctimas. Fiscalía de Pichincha, 2016-2017.}

Referente al nivel educativo, 39,7\% (171 casos) fueron bachilleres, el 35,0\% presentaron educación superior, el 17,4\% cursó la secundaria, 6,3\% presentó instrucción primaria y el $1,6 \%$ tuvo instrucción técnica.

Trabajo u ocupación de las víctimas. Fiscalía de Pichincha, 2016- 2017.

Se observa que el 40,5\% (151 casos) poseía trabajo independiente, seguido por el 35,1\% empleado privado, el 13,1\% empleado público y el 6,4\% de víctimas, indicó estar desempleado; $2,7 \%$ eran estudiantes, 1,6\% jubilados, además, cabe recalcar que la ocupación del $0,6 \%$ fueron los quehaceres domésticos

\section{- Características de la violencia, los/las agresores/as y de las agresiones.}

Relación entre la víctima y la persona agresora, según sexo. Fiscalía de Pichincha, 20162017.

El $35,3 \%$ fue la esposa (o), de los cuales 155 fueron mujeres $(35,1 \%)$ y 1 de sexo masculino $(0,2 \%)$, el $32,3 \%$ fue la conviviente $(31,7 \%$ femenino y $0,6 \%$ masculino), finalmente, del $25,8 \%$ que fueron las ex cónyuges, $25,6 \%$ fue de sexo femenino y $0,2 \%$ masculino, $6,5 \%$ de los agresores fueron las novias. 
Ocupación de los agresores. Fiscalía de Pichincha, 2016- 2017.

Se observa en su gran mayoría (37\%) 128 casos realizan quehaceres domésticos, el $24 \%$ tiene un trabajo independiente, el $17 \%$ empleado privado, el 8,4\% está desempleado o no tiene ninguna ocupación, el $7,2 \%$ fueron empleados públicos, la ocupación de estudiantes constituye el $5,8 \%$, el $0,6 \%$ fueron jubilados.

\section{- Características de las agresiones.}

Gráfico 1. Lesiones corporales de las víctimas. Fiscalía de Pichincha, 2016- 2017.

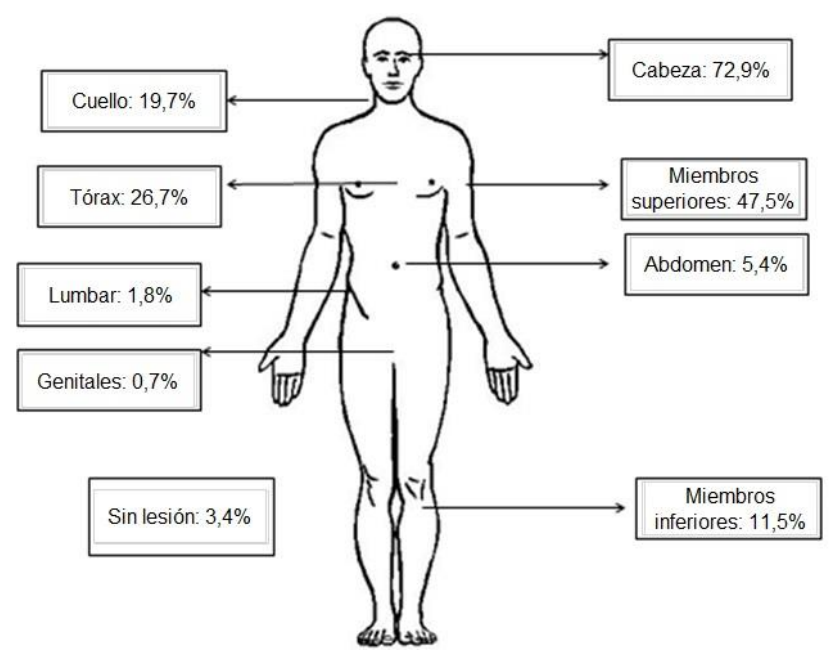

Fuente: Expedientes médico-legales de las Unidades de Atención de Peritaje Integral de Fiscalía de Pichincha.

Elaborado: Md. Mayra Viviana Barros Carvajal.

El 72,9\% (322 casos) de hombres maltratados, presentó lesiones en la cabeza/ cara, el 19,7\% ( 87 casos) en el cuello, el 26,7\% (118 casos) en el tórax, 5,4\% (24 casos) presentaron lesiones en abdomen, en los miembros superiores el 47,5\% (210 casos), el 11,5\% (51 casos) en los miembros inferiores, 3,4\% (15 casos) no presentaron lesión, además, se observó en menor porcentaje lesiones en la región lumbar ( $1,8 \%$ de los 8 casos) y en los genitales $(0,7 \%)$ en 3 casos.

Días de incapacidad médico legal acorde a las lesiones. Fiscalía de Pichincha, 20162017.

Se contó con el registro de los días de incapacidad médico legal otorgados de acuerdo con sus lesiones; de los cuales, el 3,2\% no tuvo ningún día de incapacidad, el 58,0\% de los casos tuvo menos de 3 días de incapacidad, el 21,8\% tuvo 3 días, el 15,4\% entre 4 y 8 días; mientras 
que la lesión justificó la incapacidad de 9 a 30 días en el 1,4\% de los casos y de 31 a 90 días en 1 hombre maltratado que corresponde al $0,2 \%$.

Objetos con los que recibieron el ataque de las víctimas. Fiscalía de Pichincha, 20162017.

El 3\% (13) de los 442 casos de hombres maltratados, recibieron el ataque sin ningún objeto, mientras que el $86,9 \%$ restante, sufrió el ataque con un objeto contuso.

- Frecuencia del síndrome del hombre maltratado y su relación con la violencia intrafamiliar.

Gráfico 2. Denuncias presentadas por parte de las víctimas, según casos de violencia anterior. Fiscalía de Pichincha, 2016- 2017.

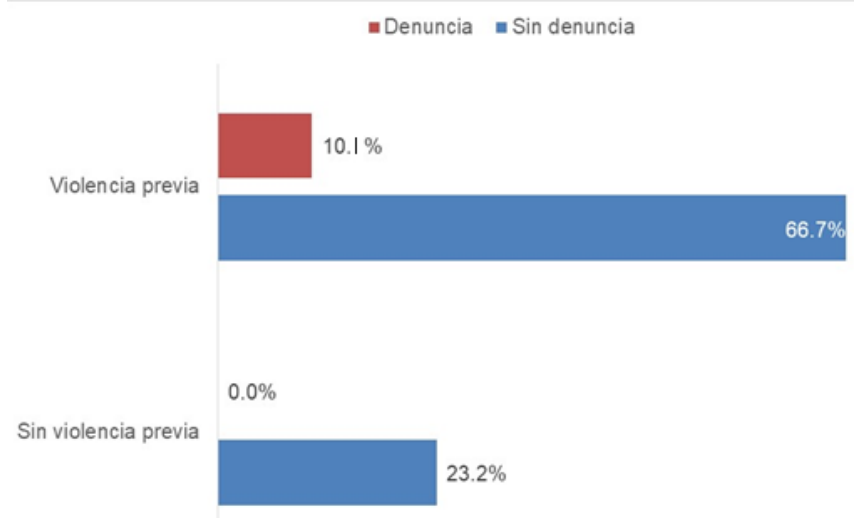

Fuente: Expedientes médico-legales de las Unidades de Atención de Peritaje Integral de Fiscalía de Pichincha.

Elaborado: Md. Mayra Viviana Barros Carvajal.

De los 442 casos de hombres maltratados, 102 (23,2\%) no sufrieron violencia anterior ni han presentado denuncias; el 66,7\% de casos vivieron violencia previa pero no realizaron ninguna denuncia; finalmente, se observa que el 10,1\% de hombres maltratados, han vivido episodios de violencia anteriormente y lo han denunciado.

Gráfico 3. Casos de violencia previa, según la relación entre el agresor y la víctima. Fiscalía de Pichincha, 2016- 2017. 


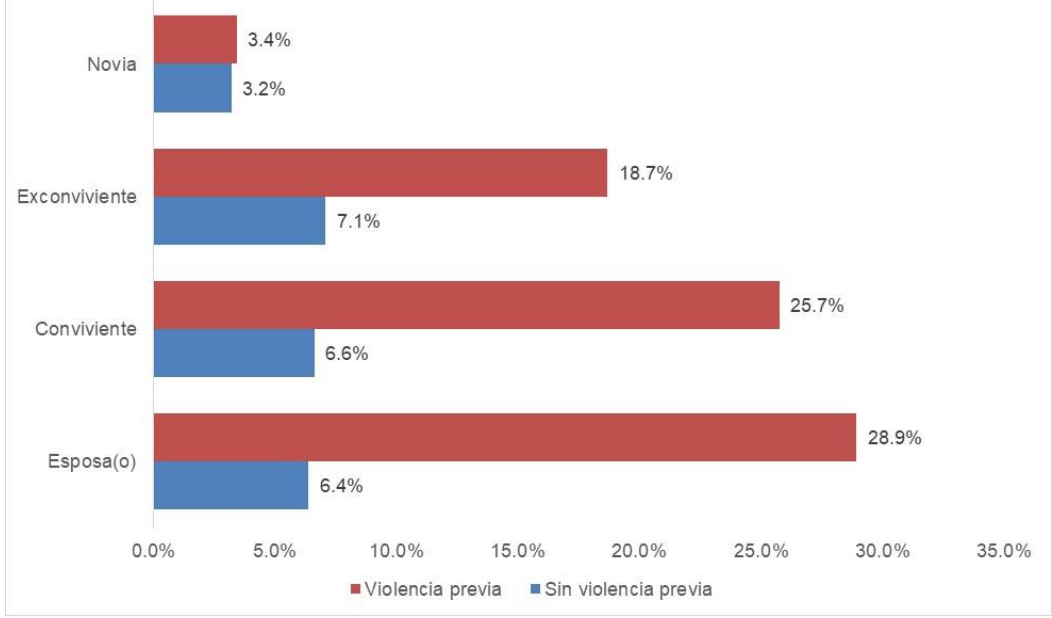

Fuente: Expedientes médico-legales de las Unidades de Atención de Peritaje Integral de Fiscalía de Pichincha.

Elaborado: Md. Mayra Viviana Barros Carvajal.

Del 76,7\% que vivió violencia previa, 127 (28,9\%) lo hizo de parte de su esposa (o), 113 $(25,7 \%)$ de parte de su conviviente, 82 de su ex conviviente $(18,6 \%$ de los 439 casos con registro de violencia previa) y 15 casos $(3,4 \%)$ de violencia previa por parte de su novia.

\section{Discusion.}

En la presente investigación se obtuvo un total de 442 datos de hombres maltratados en las dos Unidades de atención revisadas; Unidad de Flagrancia y Quitumbe, desde el primero de Enero del 2016 hasta el treinta y uno de Diciembre del 2017; se inició con la caracterización de los hombres agredidos en donde se presenta una media de edad de 35,5 años, considerando el 25,6\% de rango de edad entre 30 y 34 años el más predominante; que se puede comparar con el estudio Floyd- Aristizabal y cols en Cali del año 2014, con datos de 78 víctimas que tenían un promedio de edad de 33 años correspondiente a un 88\%; por lo tanto, la investigación presenta características similares a la descrita, donde el rango de edad entre 30 y 35 años es el porcentaje cúspide y la agresión hacia el hombre es visible.

El estado civil de las víctimas es una parte fundamental en la presente investigación, donde el $40 \%$ de agredidos estaban casados, seguidos de un 30\% que se encontraba en unión libre; mientras que en el estudio de Trujano Patricia y cols del 2010 determinó que en España (año 2000) 44 hombres fueron agredidos por parte de sus esposas, 16 casos en el 2002 y 15 en el 2005, al igual que en México el porcentaje mayor lo presentan en el estado de noviazgo más que en los casados, el estudio de Gutiérrez Natalia Colombia (20015) demostró que el 39,2\% fueron golpeados por sus esposas, el estudio Floyd- Aristizabal y cols en Cali del año 2014, indica que en el $85 \%$ de casos, fue su pareja en unión libre quien cometió la agresión y el estudio de Becerra Flores Sara y cols. Lima (2009) 53\% recibe maltrato por su esposa o 
pareja actual, demostrando que no difiere mucho con el presente estudio lo encontrado en otros países.

El nivel educativo de las víctimas el 39,7\% son bachilleres, seguida de $35 \%$ que presentan una educación superior, el 17,4\% obtuvo un nivel secundario incompleto; en el estudio de Becerra Flores Sara y cols. Lima (2009), la mayoría de hombres agredidos 58\% tiene educación superior, seguida del $42 \%$ que presenta educación secundaria; el estudio FloydAristizabal y cols en Cali del año 2014 se refiere un 49\% de varones agredidos que son bachilleres y un porcentaje menor son técnicos o tienen algún nivel educativo superior, relacionando la agresión con niveles educativos inconclusos, se encuentra el 55\% de hombres maltratados que tuvieron que abandonar sus estudios por varios motivos, entre ellos para la manutención de la familia.

En el análisis de esta investigación, se estipula que el 40,5\% de las víctimas realizan trabajo independiente, como la venta ambulante o negocios propios, con un 35,1\% de empleados privados, el $13,1 \%$ de empleados públicos, $6,4 \%$ desempleados y $0,6 \%$ que se dedican al trabajo del hogar; contraponiendo a la agresora que presenta un $37 \%$ de trabajo en el hogar, y un 24\% de trabajo independiente que por lo general es un empleo conjunto con su cónyuge, en el estudio del 2014 en Floyd- Aristizabal y cols en Cali del año 2014 y el de Gutiérrez Natalia Colombia (2015), no especifican el empleo u ocupación de las víctimas y agresores, centrándose más en el status económico y lo diferencian en diversos estratos, en el formato de la Fiscalía General del Estado para Medicina legal en Violencia Intrafamiliar ese ítem no consta por lo que se desconoce el nivel económico de la población estudiada.

Se determinó que las lesiones a nivel de cabeza y cara son un 72,9\% seguidas de los miembros superiores $47,5 \%$ y de tórax en un $26,7 \%$, de las cuales, el $76,4 \%$ fueron por lesiones contusas seguidas del $13,1 \%$ de lesiones combinadas siendo estas lesiones contusas + corto punzantes, lesiones contusas con heridas contusas, sin olvidar que las lesiones contusas las tenemos en una equimosis, un hematoma, una excoriación una laceración, el 58,0\% de las lesiones presentadas le corresponde una incapacidad menor a 3 días que se los considera contravenciones de violencia contra la mujer y miembros del núcleo familiar (art 159) acorde a nuestro Código Integral Penal; el estudio de 2014 realizado por Floyd- Aristizabal y cols en Cali señala que el 50\% de lesiones son en la piel a nivel de cara, de tipo contusas, es decir, equimosis y excoriaciones por rasguño, comparado con los datos encontrados en Quito, se encuentran con similitudes como las lesiones donde alguna parte del cuerpo es el agente lesionante; los días de incapacidad que le determinan el tiempo de privación de la libertad de cada país y sus leyes.

Finalmente de las 442 víctimas, 66,7\% presentaron violencia anterior pero el $90 \%$ no ha denunciado, siendo el $75,7 \%$ de agresoras(es) de sexo femenino, y $28,9 \%$ sus esposas con violencia previa y $6,4 \%$ de las parejas sin violencia previa; datos en común con FloydAristizabal y cols en Cali del año 2014, donde el 74\% de hombres tuvieron un maltrato 
previo de ellos el $71 \%$ fue de tipo físico y el $74 \%$ no denunció por desconocimiento y/o falta de información, según Giovanny Criollo, 2.589 delitos de violencia en Madrid-España son cometidos por mujeres, el estudio de Becerra Flores Sara y cols. Lima (2009), 26\% de la población ya no fue violentada porque decidió alejarse, el $80 \%$ de hombres que padecen maltrato, ni comenta, ni denuncia lo ocurrido, el $42 \%$ no cuenta lo sucedido por miedo a que sus hijos paguen las consecuencias; lo que refleja concordancia con los datos encontrados, correlacionándose con Colombia, España y Lima donde hay violencia previa pero sin denuncia, la violencia es física y es debida a la mujer en un gran porcentaje.

\section{Conclusiones.}

- El Síndrome de Hombre Maltratado y su relación con la violencia familiar existe y se corrobora con los 442 casos que se ha obtenido en las Unidades de Atención de Peritaje Integral de Flagrancia y de Quitumbe, donde se evidencia violencias anteriores, pero con la limitante de que no todos denuncian por el hecho de que en el Ecuador el modelo patriarcal y machismo sigue en vigencia.

- Los hombres maltratados que acudieron a Flagrancia y a las Unidades de Atención de Peritaje Integral de la ciudad de Quito entre los periodos de enero 2016 - Diciembre del 2017, fueron de estado civil casados entre 30 y 34 años edad, de escolaridad bachiller y trabajo independiente en su mayoría.

- Las características de las parejas agresoras en mayor proporción fueron sus esposas y el tipo de violencia fue la física que tuvo un alto porcentaje, con objetos de tipo contusos que determinaron una incapacidad leve, cuyo mecanismo de acción fue el uso de manos (puños), uñas (rasguños) y pies (patadas) que provocaron daños a nivel de cabeza y cara principalmente, coincidiendo con los estudios Internacionales de Colombia, España, México y Lima, cuya literatura no es amplia, pero ha sido considerable para dar certeza de la investigación realizada.

\section{Referencias bibliográficas.}

Aristizabal DLSSMIJRA. Violencia de pareja contral el hombre en Cali, Colombia. [Online].; 2016 [cited 2017 Septiembre 11. Available from: https://revistas.ucc.edu.co/index.php/ml/article/download/1700/2039

Becerra Flores Sara FVMVVJ. Violencia doméstica en contra del hombre en la ciudad de Lima. [Online]; 2009 [cited 2017 Marzo 3 [123]. Available from: https://dialnet.unirioja.es/descarga/articulo/3114291.pdf

Ecuador Rd. Código Orgánico Integral Penal. In Ecuador Rd, editor. Código Orgánico Integral Penal. Quito: Asamblea Nacional; 2013-2017. p. 1-191

Elena HP. Violencia doméstica: hombres vs mujeres maltratantes en la ciudad de Barranquilla. [Online]; 2010 [cited 2017 Marzo 3. Available from: http://www.academia.edu/27115271/Violencia_dom\%C3\%A9stica_hombres_versu s_mujeres_maltratantes_en_la_ciudad_de_Barranquilla. 
Giovani CM. DerechoEcuador.com. [Online].; 2015 [cited 2017 Marzo 3. Available from: https://www.derechoecuador.com/violenciacontraelvaron

Natalia G. Informe del evento de violencia de género, en Colombia, período epidemiológico XIII 2015. [Online].; 2015 [cited 2017 Marzo 3. Available from: http://www.medicinalegal.gov.co/documents/20143/49514/Violencia+Intrafamiliar. pdf

OMdl S. Organización Mundial de la salud. [Online].; 2017 [cited 2017 Agosto 10. Available from: http://www.who.int/topics/violence/es/.

Patricia T. Varones víctimas de violencia doméstica: Un estudio exploratorio acerca de su percepción y aceptación. [Online].; 2010 [cited 2017 Marzo 3. Available from: http://www.scielo.org.co/pdf/dpp/v6n2/v6n2a10.pdf.

Rubén RAM. Tipología del hombre víctima de la violencia conyugal. [Online]. [cited 2017 Marzo

3.

Available

from: http://repository.ucc.edu.co/bitstream/ucc/302/1/Tipolog\%C3\%ADa\%20del\%20ho mbre\%20victima\%20de\%20la\%20violencia\%20conyugal.pdf.

Shuler C. Male victims of intimate partner violence in the United States: An examination of the review of literature through the Critical theoretical persective. [Online].; 2010 [cited 2017 Marzo 3. Available from: https://www.sascv.org/ijcjs/pdfs/carolettaijcjs2010vol5iss1.pdf.

Suzanne S. The Battered husband syndrome. [Online].; 1977 [cited 2017 Enero 15. Available from: http://www.papahelp.ch/downloads/Steinmetz_The_Battered_Husband_Syndrome.p df.

Telégrafo E. El telégrafo. [Online].; 2014 [cited 2017 Febrero 4. Available from: http://www.eltelegrafo.com.ec/noticias/judicial.

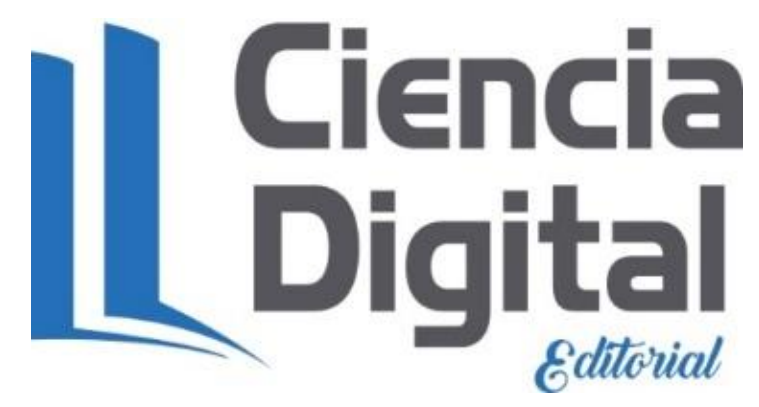


Para citar el artículo indexado.

Barros V., Chóez E. \& Zambrano F. (2019) Síndrome del hombre maltratado y la violencia intrafamiliar. Revista electrónica Ciencia Digital 3(1.1), 131-141. Recuperado desde: http://cienciadigital.org/revistacienciadigital2/index.php/CienciaDigital/article/view/365/783

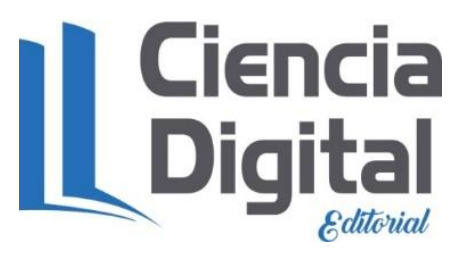

El artículo que se publica es de exclusiva responsabilidad de los autores y no necesariamente reflejan el pensamiento de la Revista Ciencia Digital.

El articulo queda en propiedad de la revista y, por tanto, su publicación parcial y/o total en otro medio tiene que ser autorizado por el director de la Revista Ciencia Digital.
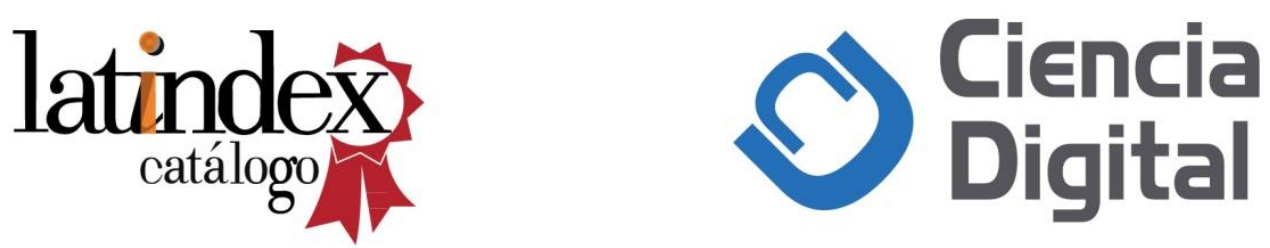